\title{
Depth enhancement in spectral domain optical coherence tomography using bidirectional imaging modality with a single spectrometer
}

Naresh Kumar Ravichandran

Ruchire Eranga Wijesinghe

Muhammad Faizan Shirazi

Kibeom Park

Mansik Jeon

Woonggyu Jung

Jeehyun Kim 


\title{
Depth enhancement in spectral domain optical coherence tomography using bidirectional imaging modality with a single spectrometer
}

\author{
Naresh Kumar Ravichandran, ${ }^{a}$ Ruchire Eranga Wijesinghe, ${ }^{a}$ Muhammad Faizan Shirazi, ${ }^{a}$ Kibeom Park, \\ Mansik Jeon, ${ }^{a, *}$ Woonggyu Jung, ${ }^{b}$ and Jeehyun Kim $^{a}$ \\ ${ }^{a}$ Kyungpook National University, School of Electronics Engineering, College of IT Engineering, 80 Daehak-ro, Buk-gu, Daegu 41566, \\ Republic of Korea \\ bUlsan National Institute of Science and Technology, Department of Biomedical Engineering, 50 UNIST-gil, Ulsan 44919, Republic of Korea
}

\begin{abstract}
A method for depth enhancement is presented using a bidirectional imaging modality for spectral domain optical coherence tomography (SD-OCT). Two precisely aligned sample arms along with two reference arms were utilized in the optical configuration to scan the samples. Using exemplary images of the optical resolution target, Scotch tape, a silicon sheet with two needles, and a leaf, we demonstrated how the developed bidirectional SD-OCT imaging method increases the ability to characterize depth-enhanced images. The results of the developed system were validated by comparing the images with the standard OCT configuration (singlesample arm setup). Given the advantages of higher resolution and the ability to visualize deep morphological structures, this method can be utilized to increase the depth dependent fall-off in samples with limited thickness. Thus, the proposed bidirectional imaging modality is apt for cross-sectional imaging of entire samples, which has the potential capability to improve the diagnostic ability. ๑2016 Society of Photo-Optical Instrumentation Engineers (SPIE) [DOI: 10 .1117/1.JBO.21.7.076005]
\end{abstract}

Keywords: optical coherence tomography; depth enhancement; bidirectional scanners; optical inspection.

Paper 160082RR received Feb. 10, 2016; accepted for publication Jun. 16, 2016; published online Jul. $11,2016$.

\section{Introduction}

Optical coherence tomography (OCT), introduced in 1991, is a noninvasive and nondestructive imaging modality that uses lowcoherence interferometry to produce high-resolution cross-sectional images. ${ }^{1}$ OCT techniques are broadly classified as time domain OCT (TD-OCT) and Fourier domain OCT (FD-OCT). FD-OCT can be further subdivided into spectral domain OCT (SD-OCT) and swept source OCT (SS-OCT). FD-OCT enables faster imaging speed with a higher signal-to-noise ratio and increased sensitivity in comparison to TD-OCT. ${ }^{2}$ Despite these numerous advantages for FD-OCT, shifting from the time domain to the Fourier domain results in some negligible artifacts, such as DC artifacts that are introduced after Fourier transformation, autocorrelation artifacts, and complex conjugate artifacts. Many techniques have been employed for the removal of these artifacts. ${ }^{3-5}$ By providing high-resolution images nondestructively and noninvasively, the applications of OCT are diverse, including areas such as in ophthalmology, dermatology, otorhinolaryngology, and agriculture. ${ }^{6-10}$ OCT has also been used in defect identification and to measure the layer thickness in electronic devices such as solar cells, LEDs, and LCDs. ${ }^{11,12}$

In FD-OCT implementations, many studies have proposed new methods and advancements to increase the OCT image resolution. Several studies have been performed to confirm the feasibility of OCT to visualize the structure and distribution of object sizes within a voxel with an ultrahigh-resolution. ${ }^{13-18}$ In addition, many research groups proposed new methods to increase the depth range, image resolution, depth of view, and field of view in OCT imaging. ${ }^{19,20}$ Especially to enhance the visible depth range, the mirror image in the OCT signal was removed by introducing an additional phase modulation and thereby performing a Hilbert transform on the OCT signal. This can be achieved either using an additional phase modulator in the reference arm setup ${ }^{21-23}$ or displacing the angle of the incident beam on the scanning mirror sample arm accordingly. ${ }^{24-26}$ Other techniques, such as producing Talbot bands before the grating in the spectrometer setup, are accomplished by shifting the interferometer beams of the backscattered signals from the reference arm and sample arm. This aids in removing the mirror terms of the OCT image and enhancing the signal-to-noise ratio. ${ }^{27,28}$ Placing the deepest part of the sample surface nearest to the zero delay and imaging will increase depth sensitivity. ${ }^{29}$ Image fusion techniques, such as Gabor fusion and zone focusing techniques, have been used with OCT to increase the depth penetration and image resolution. ${ }^{30-32}$ Furthermore, multibeam OCT techniques use multiple foci on a sample at different depths for obtaining clear images with a constant focus throughout the image. ${ }^{33}$ Computational methods have been used for solving inverse scattering and deconvolution in the out-of-focused parts of an image to increase the depth range in OCT images. ${ }^{34}$

However, a major limitation of the OCT imaging technique is that the image intensity decreases with depth. This is mostly due to broadening of the point spread function (PSF) of the coherence profile of the laser beam, which increases with imaging depth, and also because of varying refractive indices within a sample causing light reflectivity difference and thereby reducing the intensity of the reconstructed image. ${ }^{35-38}$ Many methods 
from different research groups have been developed to compensate for the broadening of the PSF and are being widely employed. ${ }^{39-44}$ Furthermore, the penetration depth of infrared light rays and the depth of focus of OCT images are also limited. Many research groups have developed new techniques to increase the depth of focus. ${ }^{45}$ Due to these limitations, the OCT imaging modality could not be effectively utilized even for samples with smaller thickness. Other research groups have demonstrated the use of multiple-directional scanning OCT. In the directional OCT method, the angle of incident light is varied in the sample arm beam and an automated hybridized chromatic mapping enables the direct visual analysis of directional information in a single image. This enables imaging of structures such as the outer nuclear layer and Henle's fiber layer thicknesses in a retina. ${ }^{46,47}$ Joint-aperture OCT (JA-OCT) is an angle-resolved OCT method where illumination from an active channel is simultaneously probed by several passive channels; thus, an increase in collection efficiency and sensitivity of the OCT system is achieved without increasing power on the sample or reducing the imaging speed. JA-OCT helps to attain a speckle reduction and increase in collection efficiency in ophthalmological imaging with OCT. ${ }^{48,49}$ All of these previous methods were used for either speckle reduction in the OCT image or for highlighting particular layers or structures in a sample. Additionally, the multidirectional scanning methods were scans from the same side of the sample.

In the conventional imaging modality setup for point scan OCT, a single-sample arm-based galvanometer scanning system has been utilized to scan the sample in order to obtain twodimensional (2-D) cross-sectional and three-dimensional (3D) volumetric images. The obtained backscattered signal is detected using a single spectrometer. The detector part of the SD-OCT system consists of an array or matrix of photosensitive elements. The backscattered OCT signal is attenuated by a sinc function in accordance with the depth, which is due to the limited pixel width of the CCD camera. Preceding research by different research groups has demonstrated methods to overcome this issue..$^{28,50,51}$ Another limiting factor in OCT imaging is the presence of mirror images that results from the Fourier transformation that is used for processing the detected OCT signal. This prohibits the maximum utilization of the detectable depth range of the CCD camera.

Unlike a conventional single sample arm-based OCT system, for samples with a small thickness and high refractive index, the proposed method enables the imaging of an entire cross-sectional thickness of the sample. This is achieved by bidirectional/dual scanning from the top and bottom surfaces of the sample. Images of the top and bottom surfaces were merged in real time with high accuracy, resulting in a cross-sectional image with enhanced depth. The developed system is most suitable for samples with thicknesses between one and two times the penetration depth of conventional single-sample armbased OCT systems, such as the fields of agronomical studies, ${ }^{52,53}$ entomological studies, ${ }^{54,55}$ and industrial inspection methods. ${ }^{56,57}$

\section{System Setup and Alignment of Bidirectional Imaging Modality}

\subsection{Optical Coherence Tomography System Setup}

A simple representation of an OCT system setup is shown in Fig. 1. The SD-OCT system is operated with a broadband light source (BroadLighters T-850-HP, Superlum) with a center wavelength of $860 \mathrm{~nm}$, a full width at half maximum of $165 \mathrm{~nm}$, and an optical power of $12.2 \mathrm{~mW}$. In total, three couplers (TW850R5A2, Thorlabs) were used to set up the optical system. All three couplers are of 50:50 ratios. The input arm of coupler- 1 is connected to the source, and each end of the output is connected to an individual sample arm along with its respective path length matched reference arm through coupler- 2 and coupler-3. The backscattered return light in the coupler arm that is adjacent to input arm has no connection to coupler- 2 and coupler-3. Coupler-2 connects with galvanometer scanner-1 (sample arm-1, which scans the top surface of the sample) and to its respective path length matched reference arm setup-1. Similarly, coupler-3 connects with galvanometer scanner-2 (sample arm-2, which scans the bottom surface of the sample) and to its respective path length matched reference setup-2. A 2-D galvanometer scanning system (GVS102, Thorlabs) is used for scanning in each sample arm setup. A 30-mm focal length lens (AC254030-B, Thorlabs) is used for focusing the beam in both of the sample arms and in both of the reference arms. The interference backscattered lights from coupler- 2 and coupler- 3 are sent back to coupler-1.

The detector part of the system is connected to the coupling arm adjacent to the source arm of coupler- 1 . The detector part is comprised of collimator-3 (LB20R, Silicon Lightwave Technology), a transmission type diffraction grating (HD1800 $\mathrm{L} / \mathrm{mm}$, Wasatch Photonics), and lens-3 of 100-mm focal length (AC508-100-B, Thorlabs), which is used for focusing the diffracted rays on a 4096-pixels line scan camera (spl4096$140 \mathrm{~km}$, Basler). The software for the built bidirectional SDOCT was programmed with compute unified device architecture (CUDA) version 3.2 for Visual studio 2008, graphics card

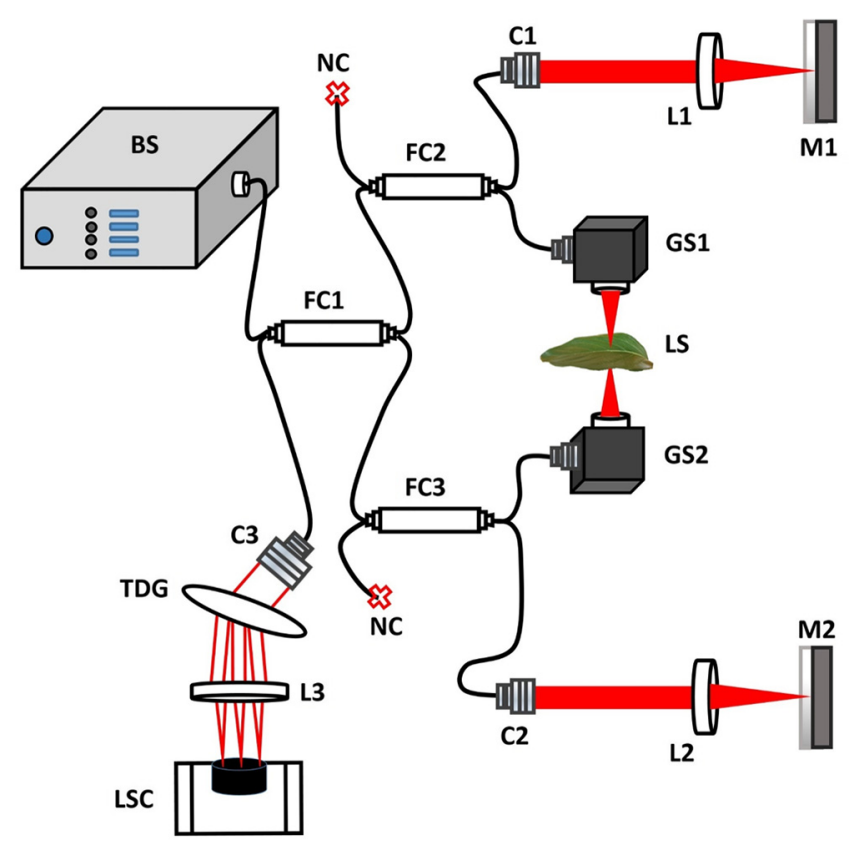

Fig. 1 Schematic diagram of the bidirectional imaging modalitybased SD-OCT system. BS is a broadband source; FC1, FC2, and FC3 are fiber couplers; $\mathrm{C} 1, \mathrm{C} 2$, and $\mathrm{C} 3$ are collimators; $\mathrm{L} 1$, L2, and L3 are lenses; M1 and M2 are mirrors; GS1 and GS2 are galvanometer scanners; LS is a leaf sample; TDG is a transmission type diffraction grating; LSC is a line scan camera; and NC means no connection. 
(Geforce GTX480, $700 \mathrm{MHz}$ Clock rate, 480 CUDA processor, NVIDIA), and CPU (Core i7 980X Hexa cores, $3.33 \mathrm{GHz}$ Clock rate, Intel). The galvanometer scanners for both sample arm-1 and sample arm-2 are driven by a personal computer (PC) with a data acquisition board (PCIe-6353, National Instruments). To attain fast data acquisition speeds, first the data acquisition thread stores the incoming 2-D signal into a buffer allocated in the random access memory in the host memory of the PC and then the signal processing thread is called. The self-repeating process continuously transfers the incoming signal to the second buffer. The frame data stored in the buffers of the host memory are copied in the device memory through the PCI express by a signal processing thread. Later, the processing divides the 480 CUDA subprocessors to further process the signal for OCT. A k-domain linearization process is carried out followed by background subtraction to remove noise in the signal. The resultant signal is Fourier transformed and log scaled to produce the 2-D OCT image. The reconstructed OCT image is then transferred back to the host memory and displayed in real-time. ${ }^{58}$ Using LabVIEW programming, the processed 2-D OCT images are obtained with a logarithmic intensity scale. The processed 2-D OCT images and the plots derived from them were initially obtained with a linear brightness scale. The brightness scale was then varied in order to best fit the image. The lateral and axial resolutions of the system were 11.4 and $3.5 \mu \mathrm{m}$ in air, respectively. A total of 500 adjacent A-scans were used to produce one 2-D image, and similarly, 500 adjacent 2-D images were processed to obtain one $3-\mathrm{D}$ image. The most probable reason for the difference between the theoretical and experimental axial resolution values can be considered to be the dispersion mismatch and chromatic aberrations caused by the optical components, taking into account that the proposed system contains comparatively more optical and additional fiber components from the conventional single-sample arm-based OCT. Although, the dispersion is compensated using numerical and hardware methods, slight wavelength-dependent loss can occur due to the optical components. Consequently, the polarization mismatch between the two interferometers leads to a loss of axial resolution as well. Similarly, the imperfect Gaussian profile of the broadband source due to the three SLDs also caused a reduction in axial resolution. The built system has a frame rate of 32 frames/s. Therefore, it takes about $31.25 \mathrm{~ms}$ to acquire one 2-D image, and for one 3-D image, i.e., 500 2-D images, it takes about $15 \mathrm{~s}$.

In the proposed bidirectional imaging method, both of the reference arm powers are maintained at half that of the maximum to maintain the near saturation level of the detector. This in turn reduces the sensitivity of the individual interferometers (interferometer- 1 comprised of reference arm- 1 and sample arm-1; interferometer-2 comprised of reference arm- 2 and sample arm-2). However, this is considered to be negligible since both of the images from the interferometers are merged to form the final OCT image, which compensates to maintain the overall sensitivity of the system.

\subsection{Alignment Method of Bidirectional Imaging Modality}

Figure 2 shows a SolidWorks 3D design, illustrating the bidirectional imaging setup for the proposed system. The entire

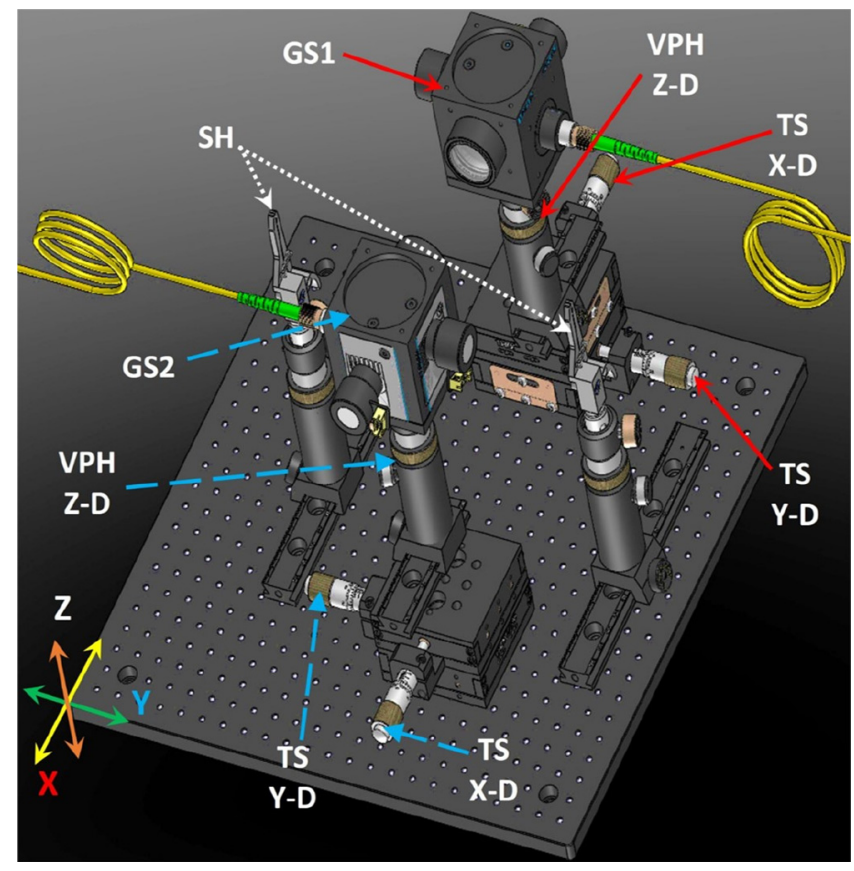

Fig. 2 SolidWorks diagram representing the bidirectional imaging setup. GS1 and GS2 are galvanometer scanners; SH is a sample holder; VPH is a variable post holder in the $z$-direction; TX is a translation stage in the $x$-direction; and TY is a translation stage in the $y$-direction. Solid red and dashed blue arrows indicate sample arm-1 and sample arm-2 components, respectively

sample arm setup is mounted on a breadboard of size $300 \mathrm{~mm} \times 300 \mathrm{~mm}$. Each galvanometer scanner is mounted on a variable post holder, a translation stage with its translating micrometer head parallel to the $x$-direction, and another translation stage with its translating micrometer head parallel to the $y$-direction. The parts of sample arm- 1 are indicated and named with red arrows in the SolidWorks design. Similarly, components incorporated for moving sample arm- 2 are indicated with blue arrows.

The image from sample arm- 2 is inverted by moving sample arm-2 toward the sample, and the path length is matched with the respective reference arm setup-2 to obtain a clearly resolved image. Aligning both sample arms to coincide their scanning positions has vital importance to avoid errors by misinterpretation of the obtained images. To overcome this, an axial and lateral resolution target (APL-OP01 OCT phantom, Arden Photonics) is used to align the sample arms scanning beam, so that the scanning point of both sample arms coincide at the same point. The refractive index of the resolution target used is 1.45. Translation stages $x$ and $y$ were used to align the sample arms in the $x$ and $y$ directions. The variable post holders were moved accordingly to match the scanning position of both sample arms in the z-direction. By using this method, the sample arms were aligned in such a way that the scanning beams from the two sample arms are aligned with micrometer accuracy. Before and after alignment in the $x, y$, and $z$ directions, the respective OCT images are shown in Figs. 3(a) and 3(b). The highlighted red box regions show the differences in intensities in the depths of the 2-D image that are taken before and after alignment.

The distance between the two sample arms can be expressed mathematically as follows: 


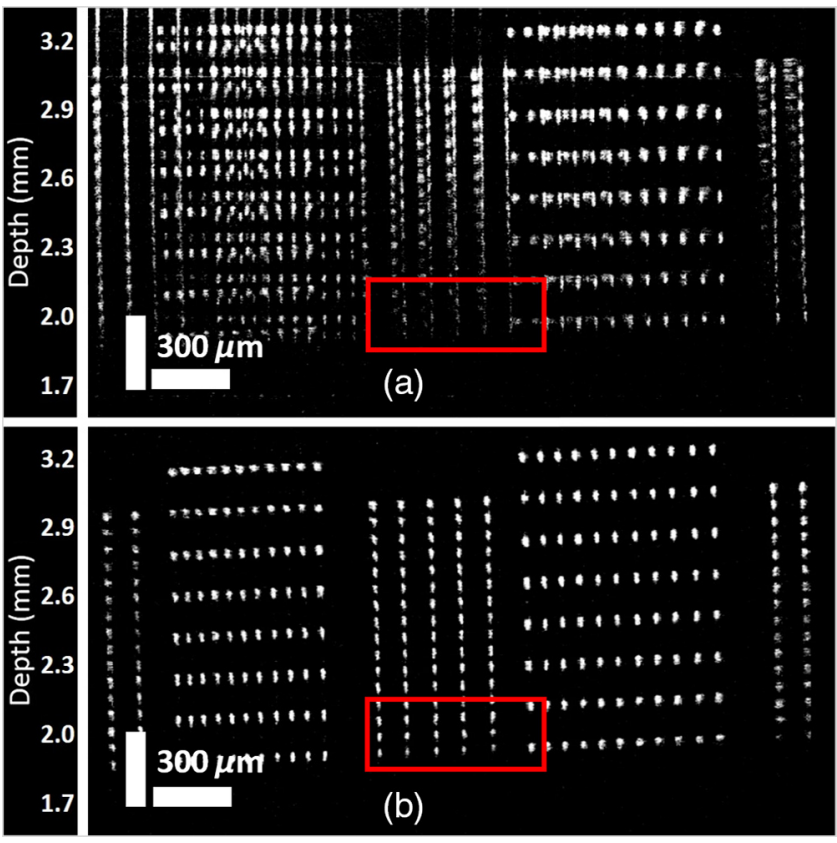

Fig. 3 Alignment of sample arms using a resolution target. (a) A 2-D cross-sectional OCT image of a lateral resolution target taken before alignment of the two sample arms. (b) The 2-D cross-sectional OCT image of a lateral resolution target taken after alignment of the two arms. The target points in the resolution target are brighter and clearly visible in (b) as compared to (a), which are highlighted in a red rectangular box.

$D_{\mathrm{L} 1 \mathrm{~L} 2}=F_{\mathrm{S} 1}+F_{\mathrm{S} 2}+T_{\mathrm{S}}-D_{\mathrm{M}}$,

where $D_{\mathrm{L} 1 \mathrm{~L} 2}$ represents the total distance between the two scanning lenses used in sample arm-1 and sample arm-2. $F_{\mathrm{S} 1}$ is the focal length of the lens used in sample arm- 1 and $F_{\mathrm{S} 2}$ is the focal length of the lens used in sample arm-2. $T_{\mathrm{S}}$ is the total sample thickness measured using a Vernier caliper, and $D_{\mathrm{M}}$ is the merged depth in the 2-D image.

The value of $D_{\mathrm{M}}$ for the above equation can be calculated using the following expression:

$D_{\mathrm{M}}=T_{\mathrm{S}}-\left[D_{\mathrm{IS} 1}+D_{\mathrm{IS} 2}\right]$.

Here, $D_{\text {IS } 1}$ is the depth of the 2-D image obtained by sample arm- 1 from the top surface of the sample and $D_{\mathrm{IS} 2}$ is the depth of 2-D image obtained by sample arm-2 from the bottom surface of the sample. $D_{\mathrm{M}}$ is the distance to be moved by translation stage- $y$ of sample arm- 2 along the $y$-axis direction toward the sample to achieve the inverted image of sample arm-2, where both images merge at the correct position.

If the path lengths of the reference arms are equal, it results in high interference. This results in a broad interference line in the OCT images. To avoid this, a minimum difference has to be maintained between the optical paths. This is represented in the following equation:

$\mathrm{NID}=\left[\left(D_{\mathrm{C} 1 \mathrm{C} 2}+D_{\mathrm{R} 1}\right)-\left(D_{\mathrm{C} 1 \mathrm{C} 3}+D_{\mathrm{R} 2}\right)\right]$,

where NID represents the noninterferometric distance to be maintained, $D_{\mathrm{C} 1 \mathrm{C} 2}$ is the distance from coupler-1 to coupler$2, D_{\mathrm{C} 1 \mathrm{C} 3}$ is the distance from coupler- 1 to coupler- $3, D_{\mathrm{R} 1}$ is the round trip propagation of light to and from coupler- 2 to the reference arm in setup- 1 , and $D_{\mathrm{R} 2}$ is the round trip propagation of light to and from coupler- 3 to the reference arm in setup-2. NID should be greater than the maximum depth ranges of the OCT system.

The total noise $\left(N_{\mathrm{T}}\right)$ occurrence in systems with multiple interferometer setups can be assessed as two types: intrainterferometric noise $\left(I_{\text {RAIN }}\right)$, which is the noise within an interferometer, and interinterferometric noise $\left(I_{\mathrm{ERIN}}\right)$, which is the noise occurrence between the interferometers.

$N_{\mathrm{T}}=\left[I_{\mathrm{RAIN}}+I_{\mathrm{ERIN}}\right]$.

The former case may occur owing to phase noise between fiber connections, optical shot noise, flicker noise in FD-OCT, Gaussian white noise, and so on. We focus on the noise occurrence between the interferometers.

In the proposed system, $I_{\text {ERIN }}$ will occur if the optical path lengths of the interferometers are the same. This may result in interference between the sample arms and reference arms of the interferometers. The following equation gives an overall representation of these noises:

$I_{\mathrm{ERIN}}=\left[I_{\mathrm{S} 1 \mathrm{~S} 2}+I_{\mathrm{R} 1 \mathrm{R} 2}+I_{\mathrm{S} 1 \mathrm{R} 2}+I_{\mathrm{S} 2 \mathrm{R} 1}\right]$,

where $I_{\mathrm{S} 1 \mathrm{~S} 2}$ refers to the noise resulting from interference between sample arm-1 and sample arm- 2 and $I_{\mathrm{R} 1 \mathrm{R} 2}$ is the noise resultant of interference between reference arm- 1 and reference arm-2. Owing to the high backscattering of the signals from the mirrors of the reference arms, this noise will contribute the highest when compared to other noise signals. $I_{\mathrm{S} 1 \mathrm{R} 2}$ is the noise interference from sample arm- 1 and reference arm-2, and $I_{\mathrm{S} 2 \mathrm{R} 1}$ is the interference from sample arm-2 and the reference arm-1 signal. During noise measurement, unused interferometer arms are blocked and only the respective interfering arm for which the noise occurrence is to be measured is kept open. The noise intensity can be estimated by taking A-scan plots for the noise signal that appears in the OCT images.

The proposed bidirectional modality has a focal plane for each sample arm. Depending upon the sample and the depth region of interest in the sample, the foci of both sample arms can be placed as needed. The foci of both sample arms can be placed at any distance from each other as long as there is no interference between the interferometers, and it depends on the depth of focus of the individual sample arms and the fall-off of the spectrometer.

\section{Results}

\subsection{Depth Enhancement Analysis Between the Bidirectional Imaging-Based SD-OCT Images over the Conventional Imaging Modality-Based SD-OCT Images}

While scanning for the conventional SD-OCT images from the top surface of the sample, both sample arm-2 and reference arm2 are blocked. Similarly, to see the bottom sample arm image separately, sample arm-1 and reference arm-1 are blocked. The physical thickness of the leaf was measured using a Vernier caliper. The total thickness of the leaf was $\sim 440 \mu \mathrm{m}$. Figures 4 (a) and 4(b) are individual sample arm images of the leaf that are taken on the adaxial and abaxial surface. Figure 4(c) is an unmerged 2-D OCT image taken with bidirectional scanning. Both of the sample arm images can be differentiated as is shown 

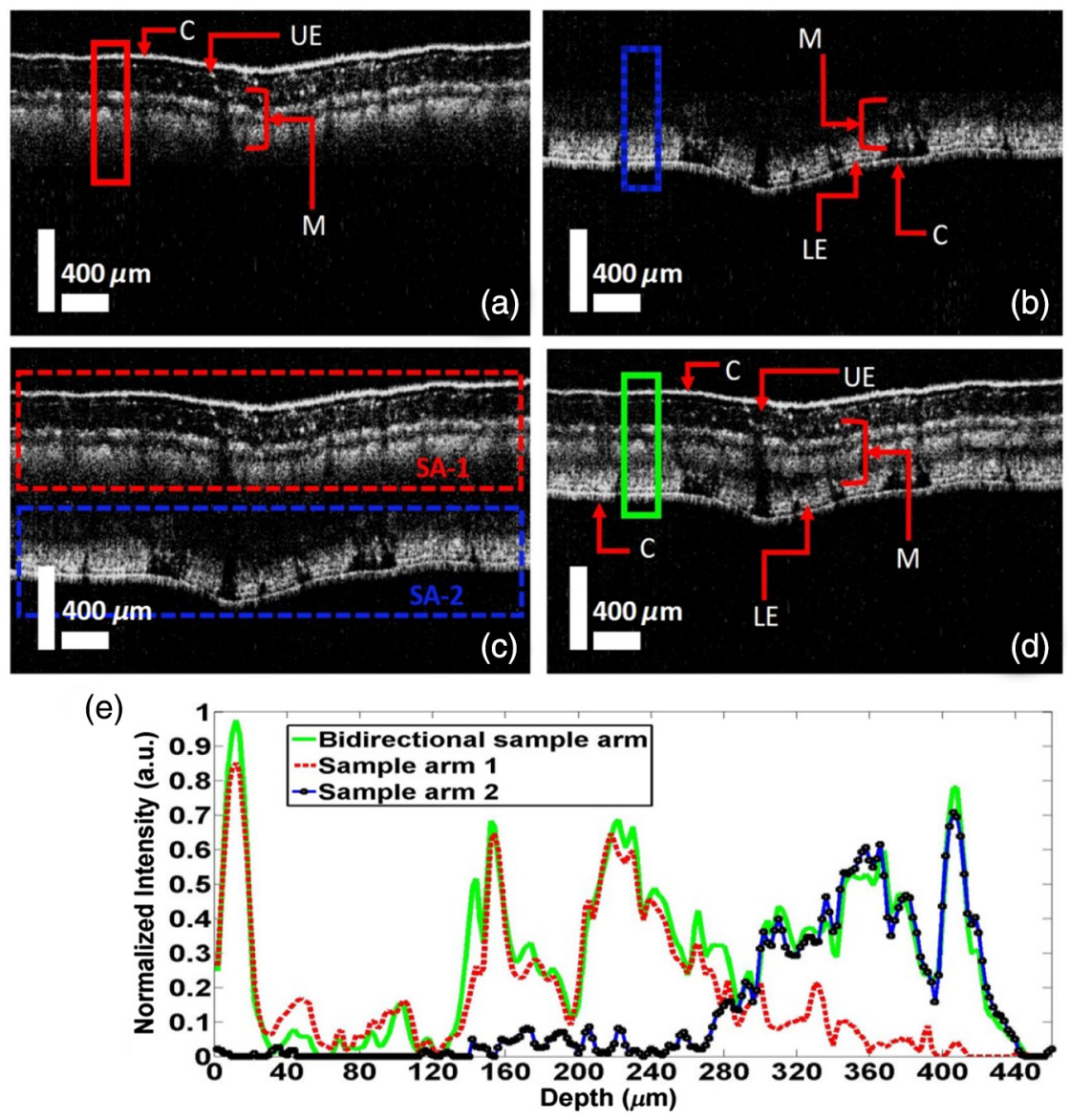

Fig. 4 Individual sample arm images along with the merged images from sample arm-1 and sample arm2. $C$ is the cuticle; UE is the upper epidermis; $M$ is the mesophyll; LE is the lower epidermis; SA-1 is the image from sample arm-1, SA-2 is the image from sample arm-2. (a) and (b) The respective 2-D crosssectional images of a leaf that was individually imaged using top sample arm-1 and sample arm-2, respectively. (c) The bidirectional sample arm image without merging. (d) The bidirectional sample arm image after merging both sample arm images. (e) The normalized A-scan plots of the individual sample arms images along with the merged bidirectional sample arm image. Rectangular boxes in red, blue, and green color in figures (a), (b), and, (d) respectively, are the regions used for A-scan analysis.

as dotted boxes in Fig. 4(c). The blue dotted box highlights the sample arm-2 image and the red dotted box highlights the sample arm-1 image. By knowing the physical thickness of the leaf measured using the Vernier caliper, the OCT images are merged accordingly. For A-scan analysis, sample regions within red, blue, and green colored boxes are used. Ten consecutive signals within these regions are normalized and the plotted graph is shown in Fig. 4(e).

Sensitivity is a function of change in refractive index and the change in optical path difference between sample and reference arm. As we know, the PSF has depth-dependent sensitivity roll off with optical path difference in the case of a mirror as a sample. ${ }^{38}$ The sensitivity profile in OCT image is dependent on the change in refractive index within the sample. The points with high-refractive index change have high sensitivity.

Figures 5(a) and 5(b) are the cross-sectional images of 7 Scotch tapes stacked one above another. The total thickness measured using a Vernier caliper was $450 \mu \mathrm{m}$. In Fig. 5(a), the middle and lower layers of the Scotch tapes are not clear. The cross-sectional image obtained using the bidirectional imaging setup gives more depth information and the layers of the sample can be seen clearly. Manually measured sample thickness is used for the alignment of the inverted sample arm-2 image by the aforementioned alignment procedure.

Figures 5(c) and 5(e) are 2-D cross-sectional images of a silicon sheet with a thickness of $1.7 \mathrm{~mm}$. The silicon sheet was impaled by two needles. The refractive index of the silicon sheet is $\sim 1.45$. A visible top layer and a marginally visible bottom layer of the silicon sheet can be seen in the conventional SD-OCT image. Furthermore, the clear visibility of both top and bottom layers of the sample using a dual-sample arm shows accurate depth enhancement. A-scan analysis plots are shown in Fig. 5(d). A set of 10 consecutive A-scans of each 2-D image were used for analyses that are highlighted in the blue and red box regions Figs. 5(a) and 5(b). The exact same area is chosen for comparative analysis. From the normalized A-scan plot, it can be seen that a total depth enhancement of $200 \mu \mathrm{m}$ is achieved in the Scotch tape sample by using the bidirectional/dual-scan imaging method over the conventional single scan image. In A-scan analysis plot, the total depth enhancement achieved (TDEA) is calculated by taking the start point of the limit at $15 \%$ or less of the intensity of the maximum peak and after that no further occurrence of higher intensity is observed. The end point is taken where a significant 

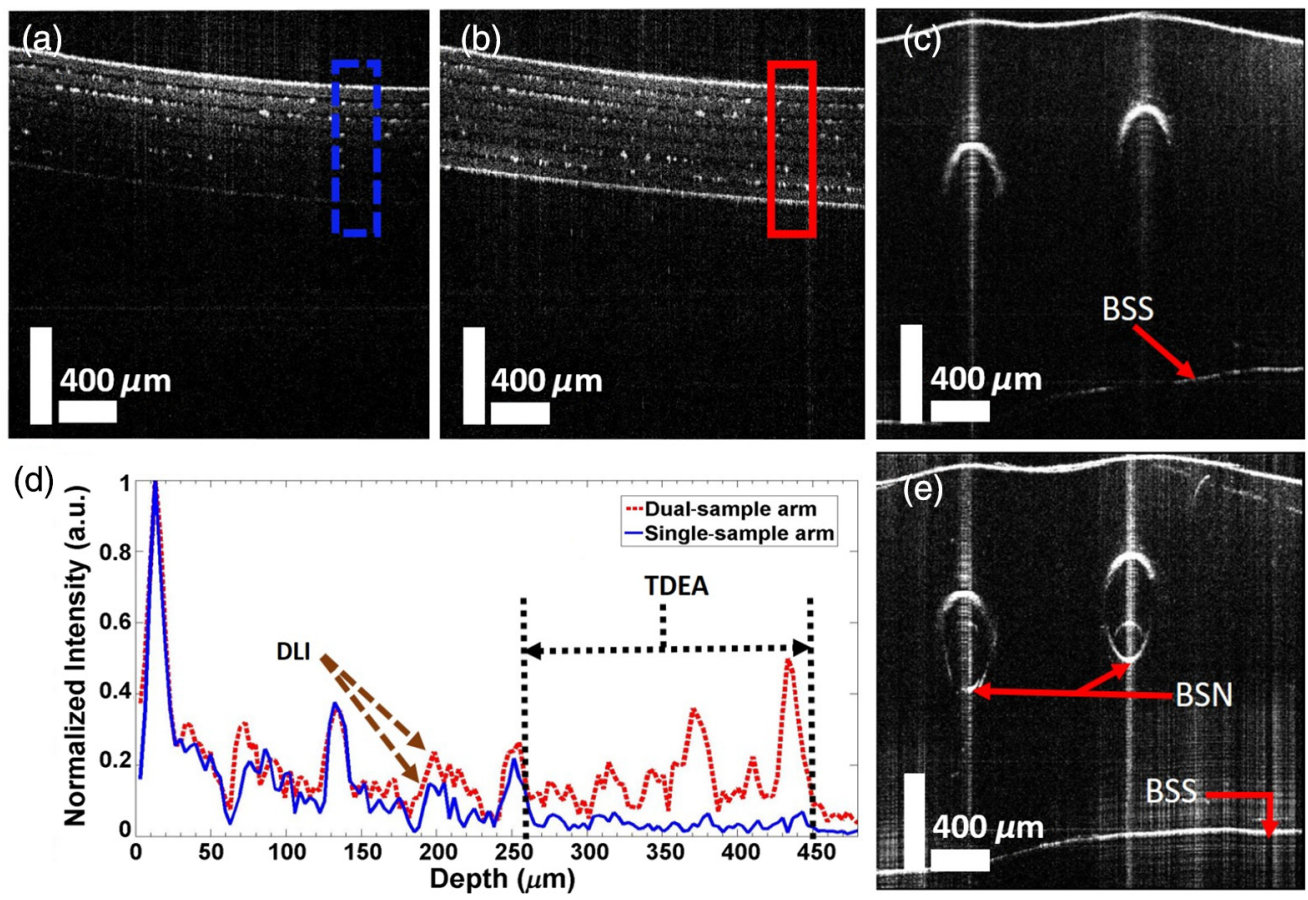

Fig. 5 Depth enhancement analysis in stacked Scotch tape and silicon sheets; BSS is the bottom surface of the silicon sheet; BSN is the bottom surface of the needle; TDEA is the total depth enhancement achieved; DLI is the difference in layer intensities. (a) and (b) The respective 2-D cross-sectional images of 7 Scotch tapes obtained with the conventional imaging modality setup and bidirectional imaging modality setup of an SD-OCT system. The blue and red box regions were used for A-scan analysis. (d) The normalized A-scan plot of (a) and (b). (c) and (e) The respective 2-D cross-sectional OCT images of a silicon sheet impaled by two needles at middle, obtained using the conventional and bidirectional SDOCT systems.

increase in intensity can be observed by the second sample arm.

\subsection{Persimmon Leaf Image Comparison Between the Bidirectional Imaging Modality and the Conventional Imaging Modality}

For image comparison between the proposed method and the conventional imaging modality, a persimmon leaf sample was utilized. Scanning was performed on the same position of the leaf using the proposed and conventional setups.

The scanning range used for both setups was $3 \mathrm{~mm} \times 3 \mathrm{~mm}$. The obtained 500 adjacent B-scan images were processed to make the 3-D images. Figure 6 images were obtained using the dual-sample arm-based SD-OCT system. Figure 6(a) shows the 3-D image and Fig. 6(c) shows the cross-sectional 2-D image. Distinguishable layers of the cuticle, upper and lower epidermis, and mesophyll are marked using red arrows in Fig. 6(c). Figure 6(b) is the orthosliced view from all three directions of axial, coronal, and sagittal. Figure $6(\mathrm{~d})$ is the enlarged view of the orthoslice in the coronal direction. The highlighted red box region shows the clear and detailed structure of the persimmon leaf. All orthoslices were at the middle position of the 3-D image.

Figure 7 was obtained using the conventional imaging modality (single-sample arm)-based SD-OCT system. Figure 7(a) shows the 3-D image, and Fig. 7(c) shows the cross-sectional 2-D image. Not all layers of the persimmon leaf are visible, unlike in Fig. 6(c). Only the cuticle in the top surface of the leaf, upper epidermis, and most of the mesophyll
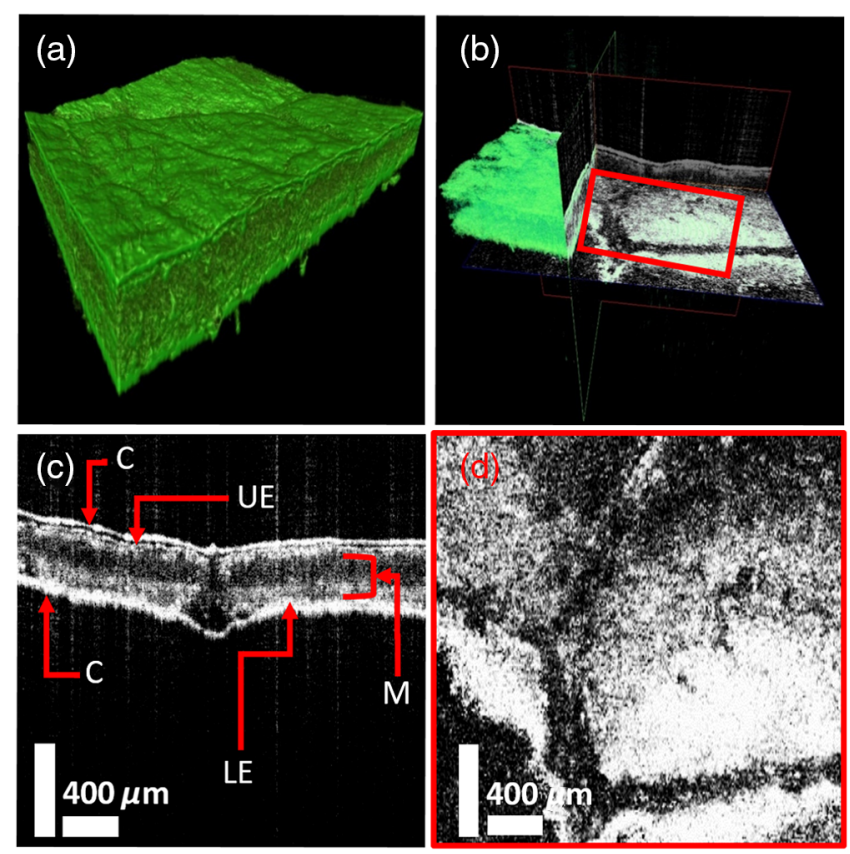

Fig. 6 Bidirectional SD-OCT images of the persimmon leaf. $C$ is the cuticle; UE is the upper epidermis; $M$ is the mesophyll; LE is the lower epidermis. (a) A 3-D image of the persimmon leaf. (b) The orthoslice of the 3-D image from all three directions (axial, coronal, and sagittal). (c) The cross-sectional 2-D image taken using the bidirectional SDOCT. (d) An enlarged view of the orthosliced image from the coronal direction. 

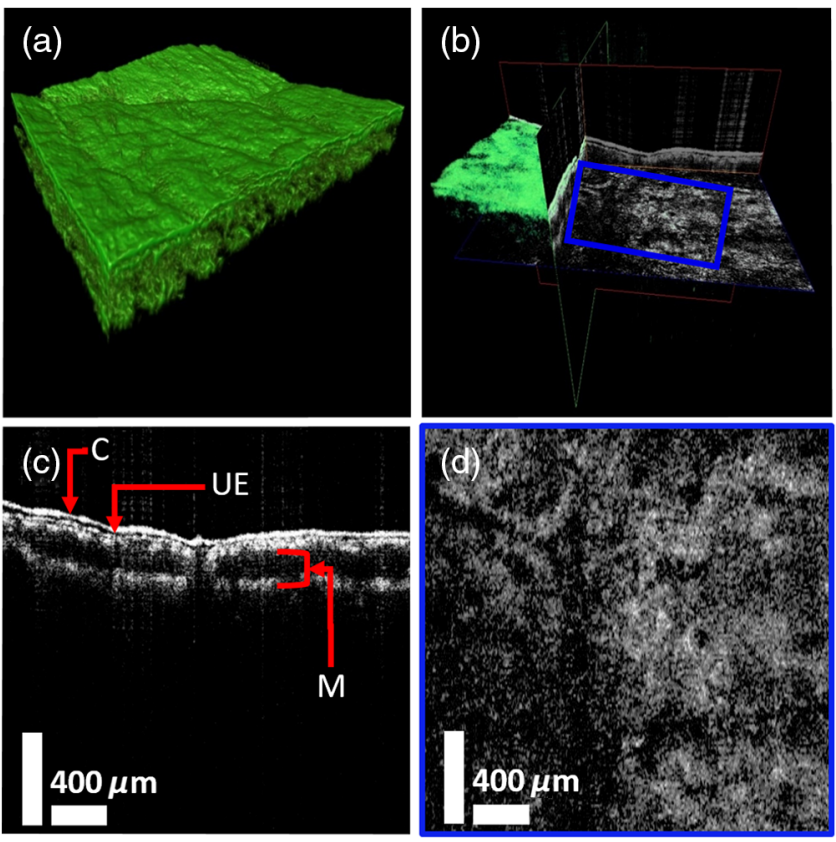

Fig. 7 Conventional single-sample arm-based SD-OCT images of the persimmon leaf. $C$ is the cuticle; UE is the upper epidermis; and $M$ is the mesophyll. (a) A 3-D image of the persimmon leaf. (b) The orthoslice of the 3-D image from all three directions [axial, coronal, sagittal are at the same position as (b)]. (c) The cross-sectional 2-D image taken using the conventional SD-OCT. (d) The enlarged view of the orthosliced image in the coronal direction and the point of orthoslice is also the same as the image taken in Fig. 6(d).

is visible in the obtained cross-sectional 2-D image using a conventional SD-OCT setup. Figure 7(b) is the orthosliced view from all three directions of axial, coronal, and sagittal. The orthoslice was at the same position as in Fig. 6(b). Figure 7(d) is the enlarged image of the orthoslice in the coronal direction, and the highlighted blue box region shows reduced intensity and the missing structural details in the mesophyll layer as compared to Fig. 6(d).

\subsection{Comparison of the A-Scan Analysis Between the Bidirectional and the Conventional SD-OCT Images}

For normalized A-scan analysis, the same location of the persimmon leaf is imaged using the bidirectional and conventional imaging modalities. In the obtained cross-sectional 2-D images, the exact same area is selected for comparative analysis. A set of 10 consecutive A-scans of each 2-D image were used for analysis. The red box region in Fig. 8(a) and blue box region in Fig. 8(b) were used for averaged A-scan analysis. The position and total area used for analysis is approximately the same in both Figs. 8(a) and 8(b).

From the obtained normalized A-scan analysis, it is clearly seen that the intensity of the peaks increases, thus the intensity fall-off with depth can be compensated by using the bidirectional imaging method. The intensity increase in layers is indicated with brown arrows in the A-scan plot. The TDEA using bidirectional imaging is about $80 \mu \mathrm{m}$ for this particular sample, which is indicated with dotted black lines in Fig. 8(c) (A-scan plot). Therefore, it is confirmed that more details and structures of the leaf layers could be identified using the bidirectional imaging method.
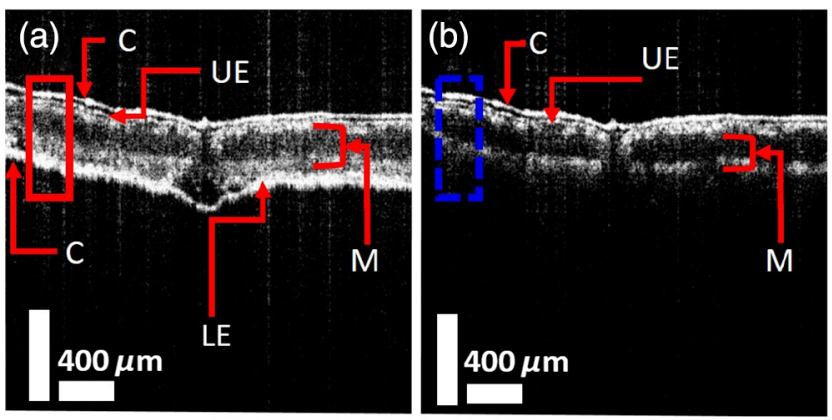

(c)

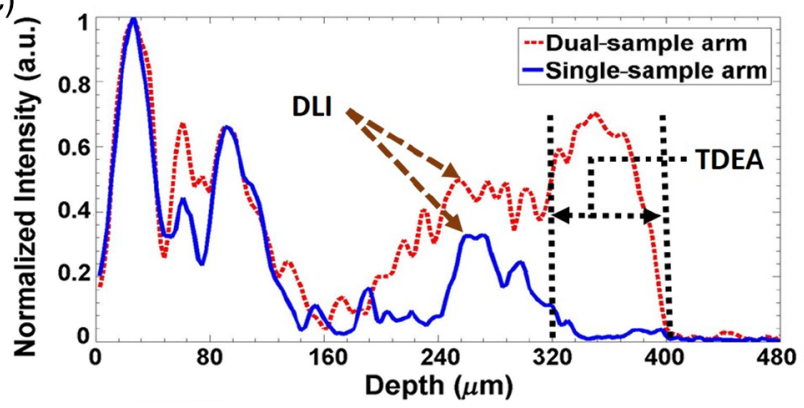

Fig. 8 Comparison of A-scan analysis of the bidirectional/dual-sample arm and the conventional single-sample arm-based SD-OCT images. $C$ is the cuticle; UE is the upper epidermis; $M$ is the mesophyll; LE is the lower epidermis; DLI is the difference in layer intensities; TDEA is the total depth enhancement achieved. (a) A 2-D cross-sectional image of the persimmon leaf taken using the bidirectional imaging SD-OCT system. The red rectangular box region indicates the area used for A-scan analysis. (b) A 2-D cross-sectional image taken in the same position of the same leaf using the conventional imaging SD-OCT system. The blue rectangular box region indicates the area used for A-scan analysis. (c) The normalized A-scan analysis plot done on (a) and (b).

\section{Conclusions}

A new approach for depth enhancement in OCT imaging using a bidirectional imaging modality with a single spectrometer has been investigated to overcome the light scattering limitation in a relatively thin sample. The proposed bidirectional imaging modality is aptly suited for samples with thicknesses between one and two times the penetration depths of conventional single-sample arm-based OCT setups. The proposed system can be utilized to its fullest in the fields of agronomical studies such as plant and seed imaging, ${ }^{52,53}$ entomological studies for in vivo studies of insects, ${ }^{54,55}$ and in industrial inspections or defect identification of samples ${ }^{56,57}$ that need entire cross-sectional imaging of samples with less thickness. The developed system provides improved depth-resolved information of the sample structures. Light scattering from deeper structures suffers from multiple scattering events resulting in a blurring effect in the region and by degradation of intensity. By employing a bidirectional scanning, an increase in intensity was observed in the middle and bottom layers of the image as well as consistent image resolution throughout the bottom layer of the specimen.

\section{Acknowledgments}

This research was supported by Advanced Production Technology Development Program (No. 314031-3) Ministry for Food, Agriculture, Forestry, and Fisheries, Republic of Korea. 


\section{References}

1. D. Huang et al., "Optical coherence tomography," Science 254(5035), 1178-1181 (1991).

2. R. Leitgeb, C. Hitzenberger, and A. F. Fercher, "Performance of Fourier domain vs. time domain optical coherence tomography," Opt. Express 11(8), 889-894 (2003).

3. M. Sarunic et al., "Instantaneous complex conjugate resolved spectral domain and swept-source OCT using $3 \times 3$ fiber couplers," Opt. Express 13(3), 957-967 (2005).

4. S. Vergnole, G. Lamouche, and M. L. Dufour, "Artifact removal in Fourier-domain optical coherence tomography with a piezoelectric fiber stretcher," Opt. Lett. 33(7), 732-734 (2008).

5. J. Ai and L. V. Wang, "Spectral-domain optical coherence tomography: removal of autocorrelation using an optical switch," Appl. Phys. Lett. 88(11), 111115 (2006).

6. M. R. Hee et al., "Quantitative assessment of macular edema with optical coherence tomography," Arch. Ophthalmol. 113(8), 1019-1029 (1995).

7. C. Pitris et al., "High-resolution imaging of gynecologic neoplasms using optical coherence tomography," Obstet. Gynecol. 93(1), 135139 (1999).

8. U. Jung et al., "Pulse analyzing system using optical coherence tomography for oriental medical application," Jpn. J. Appl. Phys. 50(5), 057001 (2011).

9. M. Jeon et al., "Methylene blue microbubbles as a model dual-modality contrast agent for ultrasound and activatable photoacoustic imaging," J. Biomed. Opt. 19(1), 016005 (2014).

10. N. K. Ravichandran et al., "In vivo monitoring on growth and spread of gray leaf spot disease in Capsicum annuum leaf using spectral domain optical coherence tomography," J. Spectrosc. 2016, 1093734 (2016).

11. S.-H. Kim, J.-H. Kim, and S.-W. Kang, "Nondestructive defect inspection for LCDs using optical coherence tomography," Displays 32(5), 325-329 (2011)

12. N. H. Cho et al., "Non-destructive inspection methods for LEDS using real-time displaying optical coherence tomography," Sensors 12(8), 10395-10406 (2012).

13. W. Drexler, "Ultrahigh-resolution optical coherence tomography," J. Biomed. Opt. 9(1), 47-74 (2004).

14. I. Hartl et al., "Ultrahigh-resolution optical coherence tomography using continuum generation in an air-silica microstructure optical fiber," Opt. Lett. 26(9), 608-610 (2001)

15. S. A. Alexandrov et al., "Nano-sensitive optical coherence tomography," Nanoscale 6(7), 3545-3549 (2014).

16. S. Aleksandrov, K. Subkhash, and M. Likhi, "Nanosensitive optical coherence tomography for the study of changes in static and dynamic structures," Quantum Electron. 44(7), 657-663 (2014).

17. M. Shirazi, W. Jung, and J. Kim, "Phase correction using programmable phase modulator (PPM) in optical coherence tomography," Biomed. Eng. Lett. 4(1), 64-72 (2014).

18. M. F. Shirazi et al., "Lateral resolution enhancement using programmable phase modulator in optical coherence tomography," Bio-Med. Mater. Eng. 26(s1), S1465-S1471 (2015).

19. J. M. Schmitt et al., "Optical-coherence tomography of a dense tissue: statistics of attenuation and backscattering," Phys. Med. Biol. 39(10), 1705-1720 (1994).

20. J. M. Schmitt, S. Xiang, and K. M. Yung, "Speckle in optical coherence tomography," J. Biomed. Opt. 4(1), 95-105 (1999).

21. R. K. Wang, "In vivo full range complex Fourier domain optical coherence tomography," Appl. Phys. Lett. 90(5), 054103 (2007).

22. J. Zhang, J. S. Nelson, and Z. Chen, "Removal of a mirror image and enhancement of the signal-to-noise ratio in Fourier-domain optical coherence tomography using an electro-optic phase modulator," Opt. Lett. 30(2), 147-149 (2005).

23. Y. Yasuno et al., "Simultaneous BM-mode scanning method for realtime full-range Fourier domain optical coherence tomography," Appl. Opt. 45(8), 1861-1865 (2006).

24. B. Baumann et al., "Full range complex spectral domain optical coherence tomography without additional phase shifters," Opt. Express 15(20), 13375-13387 (2007).

25. R. A. Leitgeb et al., "Complex ambiguity-free Fourier domain optical coherence tomography through transverse scanning," Opt. Lett. 32(23), 3453-3455 (2007).
26. L. An and R. K. Wang, "Use of a scanner to modulate spatial interferograms for in vivo full-range Fourier-domain optical coherence tomography," Opt. Lett. 32(23), 3423-3425 (2007).

27. D. Woods and A. Podoleanu, "Controlling the shape of Talbot bands' visibility," Opt. Express 16(13), 9654-9670 (2008).

28. A. Bradu and A. G. Podoleanu, "Attenuation of mirror image and enhancement of the signal-to-noise ratio in a Talbot bands optical coherence tomography system," J. Biomed. Opt. 16(7), 076010 (2011).

29. R. F. Spaide, H. Koizumi, and M. C. Pozonni, "Enhanced depth imaging spectral-domain optical coherence tomography," Am. J. Ophthalmol. 146(4), 496-500 (2008).

30. J. P. Rolland et al., "Gabor-based fusion technique for optical coherence microscopy," Opt. Express 18(4), 3632-3642 (2010).

31. P. Bouchal, A. Bradu, and A. G. Podoleanu, "Gabor fusion technique in a Talbot bands optical coherence tomography system," Opt. Express 20(5), 5368-5383 (2012).

32. W. Drexler et al., "In vivo ultrahigh-resolution optical coherence tomography," Opt. Lett. 24(17), 1221-1223 (1999).

33. B. A. Standish et al., "In vivo endoscopic multi-beam optical coherence tomography," Phys. Med. Biol. 55(3), 615-622 (2010).

34. G. Liu et al., "Automatic estimation of point-spread-function for deconvoluting out-of-focus optical coherence tomographic images using information entropy-based approach," Opt. Express 19(19), 1813518148 (2011).

35. G. Tearney et al., "Determination of the refractive index of highly scattering human tissue by optical coherence tomography," Opt. Lett. 20(21), 2258-2260 (1995).

36. J. M. Schmitt, "Optical coherence tomography (OCT): a review," IEEE J. Sel. Top. Quantum Electron. 5(4), 1205-1215 (1999).

37. A. Knu and M. Boehlau-Godau, "Spatially confined and temporally resolved refractive index and scattering evaluation in human skin performed with optical coherence tomography," J. Biomed. Opt. 5(1), 83$92(2000)$

38. M. Jeon et al., "Full-range k-domain linearization in spectral-domain optical coherence tomography," Appl. Opt. 50(8), 1158-1163 (2011).

39. M. Mujat et al., "Autocalibration of spectral-domain optical coherence tomography spectrometers for in vivo quantitative retinal nerve fiber layer birefringence determination," J. Biomed. Opt. 12(4), 041205 (2007).

40. Z. Wang et al., "Increasing the imaging depth of spectral-domain OCT by using interpixel shift technique," Opt. Express 14(16), 7014-7023 (2006).

41. B. C. Lee, M. Y. Jeon, and T. J. Eom, "k-domain linearization of wavelength-swept laser for optical coherence tomography," Proc. SPIE 7894, 789418 (2011).

42. Z. Hu and A. M. Rollins, "Fourier domain optical coherence tomography with a linear-in-wavenumber spectrometer," Opt. Lett. 32(24), 3525-3527 (2007).

43. U.-S. Jung et al., "Simple spectral calibration method and its application using an index array for swept source optical coherence tomography," J. Opt. Soc. Korea 15(4), 386-393 (2011).

44. S. M. Kolenderska, G. Wilczyński, and M. Wojtkowski, "Blue-light Fourier-domain optical-coherence microscopy with linear k-sampling using second-harmonic generation," Opt. Lett. 40(15), 3540-3543 (2015).

45. Z. Ding et al., "High-resolution optical coherence tomography over a large depth range with an axicon lens," Opt. Lett. 27(4), 243-245 (2002).

46. V. S. Makhijani et al., "Chromatic visualization of reflectivity variance within hybridized directional OCT images," Proc. SPIE 8571, 857105 (2013).

47. B. J. Lujan et al., "Revealing Henle's fiber layer using spectral domain optical coherence tomography," Invest. Ophthalmol. Visual Sci. 52(3), 1486-1492 (2011).

48. T. Klein et al., "Joint aperture detection for speckle reduction and increased collection efficiency in ophthalmic MHz OCT," Biomed. Opt. Express 4(4), 619-634 (2013).

49. M. Hughes, M. Spring, and A. Podoleanu, "Speckle noise reduction in optical coherence tomography of paint layers," Appl. Opt. 49(1), 99107 (2010).

50. B. Grajciar et al., "Parallel Fourier domain optical coherence tomography for in vivo measurement of the human eye," Opt. Express 13(4), 1131-1137 (2005). 
51. T. Bajraszewski et al., "Improved spectral optical coherence tomography using optical frequency comb," Opt. Express 16(6), 4163-4176 (2008).

52. S.-Y. Lee et al., "Application of optical coherence tomography to detect cucumber green mottle mosaic virus (CGMMV) infected cucumber seed," Hortic. Environ. Biotechnol. 53(5), 428-433 (2012).

53. C.-H. Lee et al., "The application of optical coherence tomography in the diagnosis of Marssonina blotch in apple leaves," J. Opt. Soc. Korea 16(2), 133-140 (2012).

54. M. Kambe et al., "In-vivo imaging of developing wings in butterfly pupa by using optical coherence tomography," J. Korean Phys. Soc. 53(2), 1290-1294 (2008).

55. K. Brown and M. Harvey, "Optical coherence tomography: age estimation of Calliphora vicina pupae in vivo?" Forensic Sci. Int. 242, 157161 (2014).

56. K. Wiesauer et al., "Investigation of glass-fibre reinforced polymers by polarisation-sensitive, ultra-high resolution optical coherence tomography: Internal structures, defects and stress," Compos. Sci. Technol. 67(15), 3051-3058 (2007).
57. P. Targowski et al., "Structural examination of easel paintings with optical coherence tomography," Acc. Chem. Res. 43(6), 826-836 (2010).

58. N. H. Cho et al., "Quantitative assessment of touch-screen panel by nondestructive inspection with three-dimensional real-time display optical coherence tomography," Opt. Lasers Eng. 68, 50-57 (2015).

Mansik Jeon received his $\mathrm{PhD}$ in electronics engineering from Kyungpook National University, Daegu, Republic of Korea, in 2011. Currently, he is an assistant professor of the School of Electronics Engineering at Kyungpook National University. His research interests are in the development of nonionizing and noninvasive novel biomedical imaging techniques, including photo acoustic tomography, optical coherence tomography, ultrasonic imaging, handheld scanners, and their clinical applications.

Biographies for the other authors are not available. 